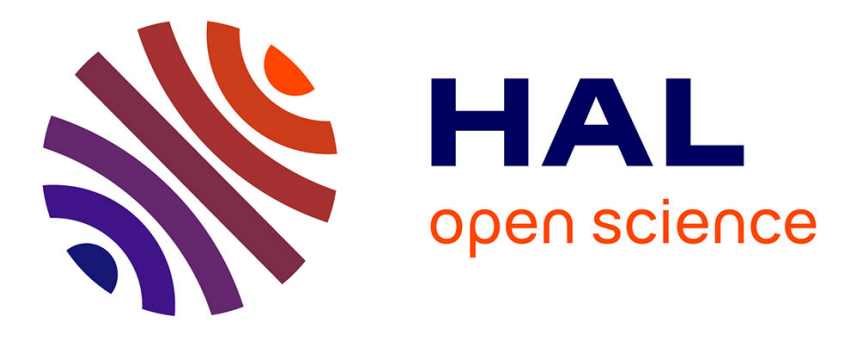

\title{
Photomodulation of DNA-Templated Supramolecular Assemblies
}

Jenifer Rubio-Magnieto, Tuan-Anh Phan, Mathieu Fossépré, Valérie Matot, Jeremie Knoops, Thibaut Jarrosson, Pascal Dumy, Françoise Serein-Spirau, Claude Niebel, Sébastien Ulrich, et al.

\section{To cite this version:}

Jenifer Rubio-Magnieto, Tuan-Anh Phan, Mathieu Fossépré, Valérie Matot, Jeremie Knoops, et al.. Photomodulation of DNA-Templated Supramolecular Assemblies. Chemistry - A European Journal, 2018, 24, pp.706-714. 10.1002/chem.201704538 . hal-01666843

\section{HAL Id: hal-01666843 https://hal.science/hal-01666843}

Submitted on 25 May 2021

HAL is a multi-disciplinary open access archive for the deposit and dissemination of scientific research documents, whether they are published or not. The documents may come from teaching and research institutions in France or abroad, or from public or private research centers.
L'archive ouverte pluridisciplinaire HAL, est destinée au dépôt et à la diffusion de documents scientifiques de niveau recherche, publiés ou non, émanant des établissements d'enseignement et de recherche français ou étrangers, des laboratoires publics ou privés. 


\title{
Photomodulation of DNA-templated supramolecular assemblies
}

\author{
Jenifer Rubio-Magnieto, ${ }^{[a]}$ Tuan-Anh Phan, ${ }^{[b, c]}$ Mathieu Fossépré, ${ }^{[a]}$ Valérie Matot, ${ }^{[a]}$ Jérémie Knoops, ${ }^{[a]}$ \\ Thibaut Jarrosson, ${ }^{[b]}$ Pascal Dumy, ${ }^{[c]}$ Françoise Serein-Spirau, ${ }^{[b]}$ Claude Niebel, ${ }^{*[b]}$ Sébastien Ulrich, ${ }^{*[c]}$ \\ and Mathieu Surin ${ }^{*[a]}$
}

\begin{abstract}
A new type of DNA ligand that contains a phosphatebinding group and a photoresponsive azobenzene moiety is reported When the azobenzene is in trans configuration, the ligand binds to the minor groove of a double-stranded DNA, whereas it partially desorbs upon trans-cis isomerization with light. The ability to photoswitch the ligand upon interaction with DNA is evidenced by (chir)optical signatures, and deciphered by the differences of binding geometry, stability, and dynamics of the DNA/ligand complexes for the two isomers. We exploit these properties to photomodulate DNAtemplated self-assembly, through the incorporation of another $\pi$ stacking DNA ligand, which together with the photoresponsive ligand form mixed supramolecular complexes along DNA. Our study demonstrates that well-designed photo-responsive DNA binders can be used to modulate multicomponent supramolecular DNA assemblies.
\end{abstract}

\section{Introduction}

The fine control over the formation of supramolecular assemblies and polymers via DNA templating has recently gained great interest to achieve functional nanostructures, with possible applications in sequence-controlled polymers, (bio)sensing, and delivery. ${ }^{[1-11]}$ In this regard, achieving DNAtemplated multicomponent assemblies for which the organization and function can be modulated upon external stimuli is a fascinating challenge. ${ }^{[6,12-18]}$ Among the different stimuli, light is a practical trigger for enabling responsive materials, ${ }^{[13,17]}$ as exemplified by the incorporation of photoswitchable ligands into biomolecular systems in view of

[a] Dr. J. Rubio-Magnieto, Dr. M. Fossépré, V. Matot, J. Knoops, Prof. M. Surin

Laboratory for Chemistry of Novel Materials

Center of Innovation and Research in Materials and Polymers

University of Mons - UMONS,

20 Place du Parc, 7000 Mons, Belgium

E-mail: mathieu.surin@umons.ac.be

[b] T.-A. Phan, Dr. T. Jarrosson, Prof. F. Serein-Spirau, Dr. C. Niebel Institut Charles Gerhardt, UMR 5253, CNRS, Université de Montpellier

ENSCM, Ecole Nationale Supérieure de Chimie de Montpellier, 240 Avenue du Professeur Emile Jeanbrau, 34296 Montpellier cedex 5, France. E-mail: claude.niebel@enscm.fr

[c] T.-A. Phan, Prof. P. Dumy, Dr. S. Ulrich Institut des Biomolécules Max Mousseron (IBMM) UMR 5247 CNRS, Université de Montpellier, ENSCM,

Ecole Nationale Supérieure de Chimie de Montpellier, 240 Avenue du Professeur Emile Jeanbrau, 34296 Montpellier cedex 5, France.

E-mail: sebastien.ulrich@enscm.fr.

Supporting information for this article is given via a link at the end of the document. controlling their conformation and activity in a reversible manner. ${ }^{[12]}$ Several groups have already developed photoresponsive DNA molecules by covalently linking photoswitching molecules within the nucleic acid structure (e.g. azobenzenes in between base pairs). ${ }^{115,19-27]}$ This covalent approach permits the formation and dissociation of DNA duplex by illuminating the azo-modified oligonucleotide with UV light, which is valuable for the photoregulation of bioreactions. ${ }^{[19,22,28}$ 29] However, the main drawback of this incorporation is the difficulty in synthesis/preparation of these covalently modifiedDNA, which restricts its use. In this context, the use of photoswitchable molecules that can interact with the DNA via supramolecular recognition offers an alternative approach. ${ }^{[24,30]}$ For instance, spyropyran or azobenzene derivatives were used as photosensitive DNA intercalators, and the use of an adequate light source permitted to switch between association/dissociation of the intercalator with DNA or between assembly/disassembly of DNA nanostructures. ${ }^{\text {[1-32] }}$

In this article, we report on the design and characterization of a photoswitch (Gua-Azo, Chart 1) containing a guanidinium group, which is purposeful for DNA binding and has been shown to be valuable for applications in DNA sensing and gene delivery. ${ }^{[25,33-42]}$ The photoswitch is brought by an azobenzene moiety, which can undergo a reversible photoisomerization with UV light $(365 \mathrm{~nm}$, trans $\rightarrow$ cis, E/Z) and visible light $(450 \mathrm{~nm}$, cis $\rightarrow$ trans, $Z / E)$.

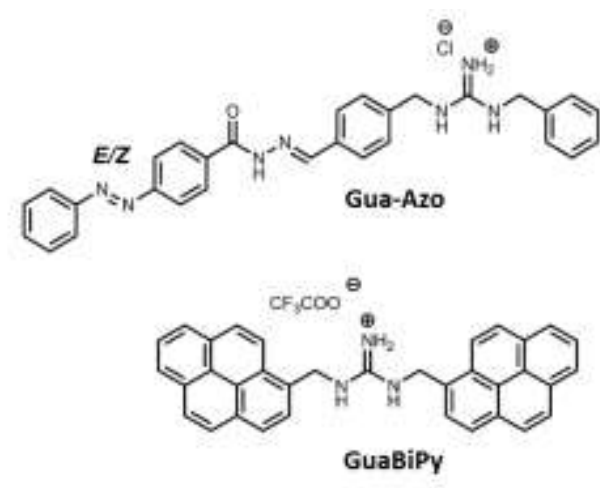

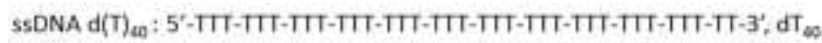
dsDNA $d(R)_{20} d(R)_{10}$ 20: 5'-CGT CAC GTA AAT CGG TTA AC - $3^{\prime}$ 3'-GCA GTG CAT TTA GCC AAT TG-5; ds 20

stONA : salmon DNA, -2000 base pairs

Chart 1. Top: Chemical structures of Gua-Azo photoswitch and GuaBiPy ligand. Bottom: DNA templates used in this study.

tas

$>$

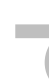


We demonstrate that the binding of this photoswitch molecule along the DNA can be modulated by light: when the azobenzene is in trans conformation, Gua-Azo binds DNA and show induced chiroptical signals, whereas the cis conformation has a lower affinity and show no induced chiroptical signal. This is rationalized by molecular modelling simulations, showing that the trans isomer perfectly accommodate into the DNA minor-groove of double-stranded DNA (dsDNA), whereas the geometry of the cis leads to partial desorption of the azobenzene moiety from DNA minorgroove, a lower stability and different dynamics of the complex. Moreover, the photoresponsive DNA binder Gua-Azo can form mixed assemblies with another guanidinium-based compound such as GuaBiPy (Chart 1), which permits the photomodulation of the supramolecular assembly along the DNA strands and therefore represents a step forward toward the photo-control of multicomponent DNA-templated selfassemblies.

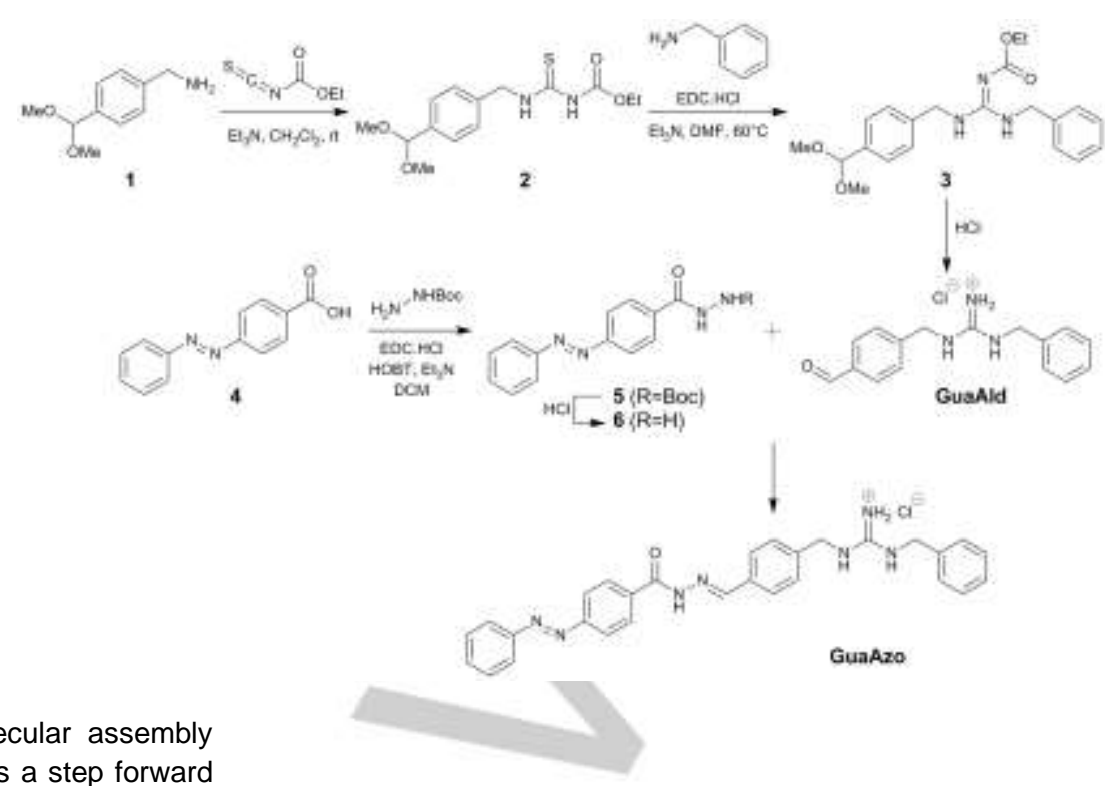

Scheme 1. Synthetic route for the preparation of compound Gua-Azo.

\section{Results and Discussion}

\section{Design and synthesis of Gua-Azo}

We have previously reported the supramolecular self-assembly of GuaBiPy (Chart 1) along single-stranded and doublestranded DNA templates. ${ }^{[11,43]}$ This initial study revealed that, in addition to salt-bridge interactions between the guanidinium groups and the DNA phosphodiesters, $\pi$-stacking interactions are essential for stabilizing these DNA-templated complexes. We reasoned that the insertion of an azobenzene photoswitch would enable the photocontrol of this type of supramolecular assemblies given that the $E$ isomer (trans) is planar and should therefore stabilize the assemblies, whereas the $Z$ isomer (cis) is forced to be non-planar, which should destabilize the assembly.

Based upon our previous experience in using acylhydrazone coupling reactions for synthesizing bioconjugates, ${ }^{[43,44]}$ we selected a strategy that involves preparing Gua-Azo compound through the reaction between aldehyde GuaAld and the azohydrazide 5 (Scheme 1 and Figs. S1-S13 in the Supporting Information). GuaAld was prepared in three steps involving: i) the formation of thiourea 2 by addition of 4-(dimethoxymethyl)benzylamine $\mathbf{1}$ onto ethoxycarbonyl isothiocyanate; ii) the subsequent EDC-mediated coupling with benzylamine to afford compound $\mathbf{3}$, and iii) the hydrolysis of the ketal and carbamate protecting groups. 4-(phenylazo) benzohydrazide 6 was obtained in two steps: peptide coupling between 4-(phenylazo)benzoic acid 4 and tert-butyl carbazate affords compound $\mathbf{5}$ which was deprotected in acidic conditions to furnish the desired hydrazide $\mathbf{6}$. Finally, compounds GuaAld and 6 are combined to yield after acylhydrazone ligation the desired Gua-Azo in a $40 \%$ isolated yield.

\section{Photoisomerization of Gua-Azo}

The photoisomerization experiments were carried out using a LED source by illuminating a solution of Gua-Azo compound ( $120 \mu \mathrm{M})$ at $365 \mathrm{~nm}$ during 5 minutes, yielding the trans $\rightarrow$ cis $(E / Z)$ isomerization of the azobenzene moiety. The UV-Vis spectra (Figure 1) showed a decrease of the initial $\pi-\pi^{*}$ absorption band at $330 \mathrm{~nm}$ with a large blue shift $(\sim 20 \mathrm{~nm})$ and a weak increase of the $n-\pi^{*}$ absorption band at around $430 \mathrm{~nm}$, narrowed compared to the absorption of the trans isomer in this wavelength range (the $n-\pi^{*}$ electronic transition is only allowed for the cis isomer). ${ }^{[20,25]}$ Under illumination of the solution at 450 $\mathrm{nm}$, we observed the recovery of signals of the trans form, both on the intensity of the $\pi-\pi^{*}$ transition and the decrease of the $n$ $\pi^{*}$ absorption band, thereby demonstrating the reversible and photocontrolled cis $\rightarrow$ trans $(Z / E)$ isomerization.

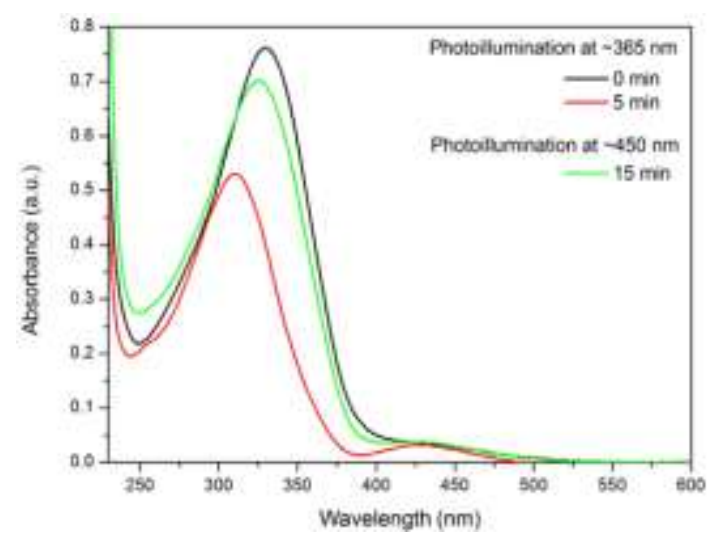

Figure 1. UV-Vis absorption spectra of Gua-Azo $(\sim 120 \mu \mathrm{M})$ in Milli-Q water upon illumination. [Gua-Azo] $]_{\text {stock }}=10 \mathrm{mM}$ in DMSO. 
${ }^{1} \mathrm{H}$ NMR studies in $d_{6}$-DMSO also confirmed the reversible photoswitch of Gua-Azo compound. Upon irradiation at $365 \mathrm{~nm}$ complete conversion of the trans isomer into the cis isomer takes place within $15 \mathrm{~min}$ and the subsequent cis $\rightarrow$ trans backisomerization was achieved by excitation of the $n-\pi^{*}$ absorption band (Fig. S14).

\section{Photoisomerization of Gua-Azo in presence of DNA}

DNA binding of trans isomer of Gua-Azo. The interactions between Gua-Azo and DNA were studied by UV-Vis and Circular Dichroism (CD) at different molar ratios at $20^{\circ} \mathrm{C}$. For that, Gua-Azo in its stable trans $(E)$ configuration was mixed with selected DNA, either with short oligonucleotides (ODN, $\mathrm{dT}_{40}$ or ds $\mathrm{R}_{20}$ ) or long genomic DNA (salmon sperm DNA, stDNA), see Chart 1. With a homopyrimidine single-stranded DNA dT $\mathrm{T}_{40}$, the CD spectra show the characteristic CD bands of DNA, centred at $276 \mathrm{~nm}$ and $250 \mathrm{~nm}$, which are less intense upon the addition of Gua-Azo (see Figure 2). Interestingly, an induced circular dichroism (ICD) appears in the 300-400 nm range (see red line in Figure 2b), i.e. where the Gua-Azo absorbs albeit the pure compound is CD-silent (Fig. S15-S16).

This positive ICD signal shows a maximum intensity at a molar ratio around 1:50 in $\mathrm{dT}_{40}$ :Gua-Azo (Fig. S15), showing that the Gua-Azotrans adopts a chiral organization upon DNA binding. Note that ICD signals of ligands upon DNA binding were previously reported for other guanidinium derivatives, ${ }^{[11,32,36]}$ including GuaBiPy for which the maximum ICD is around 1:20 in $\mathrm{dT}_{40}$ :GuaBiPy (single-stranded DNA template). ${ }^{[11]}$ The same experiments were repeated on double-stranded DNAs (dsDNA), a duplex oligonucleotide ( $d s R_{20}$ ) and a long dsDNA (stDNA). Upon addition of Gua-Azo with each of the dsDNA, important positive ICD signals also appear in the $300-400 \mathrm{~nm}$ region (Figure 2d,f), similar to that observed with the ssDNA. However, for the long DNA, we observed a bisignate (-/+) ICD signal in the Gua-Azo region with a zero crossing at around $403 \mathrm{~nm}$.

DNA binding of cis isomer of Gua-Azo. We added a solution of DNA right after having UV illuminated a Gua-Azo solution under a LED (working wavelength at around $365 \mathrm{~nm}$ ), i.e. yielding a large proportion of Gua-Azo in cis form. The UV-Vis absorption spectra of the [Gua-Azocis - DNA] mixture (see green lines in Figure 2) show a decreased intensity of the $\pi-\pi^{*}$ band and a change in the absorption maxima towards lower wavelengths $\left(\lambda_{\max } \sim 313 \mathrm{~nm}\right)$, which corresponds to the $\lambda_{\max }$ of the Gua-Azo in cis configuration $(\sim 310 \mathrm{~nm})$. Importantly, we observe the appearance of a weaker $n-\pi^{*}$ absorption band in the visible region ( $429 \mathrm{~nm}$ ), as shown with pure Gua-AzOcis. The $\mathrm{CD}$ spectra of complexes with oligonucleotides (either $\mathrm{dT}_{40}$ or $\mathrm{dsR}_{20}$ ) show no ICD signal in the range where the Gua-Azo absorbs (see Figure $2 b, d$ ), which indicates that the cis conformer does not adopt a preferential chiral organization upon binding with the short DNA. In contrast, with the long dsDNA (stDNA), an ICD bisignate signal is observed (Figure 2f) but weaker than that for the trans form.

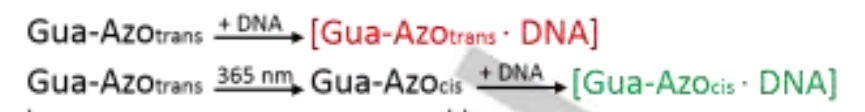
a)
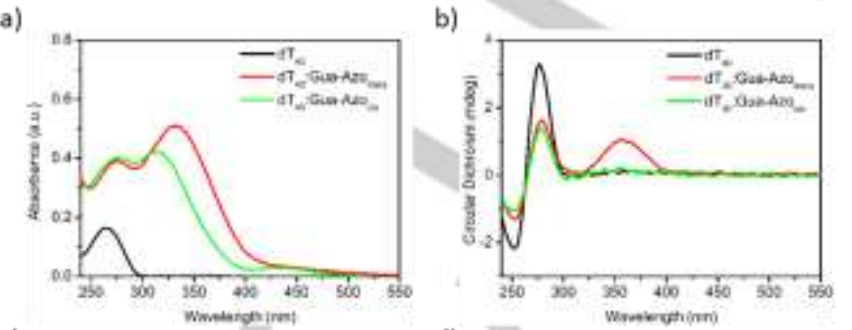

c)
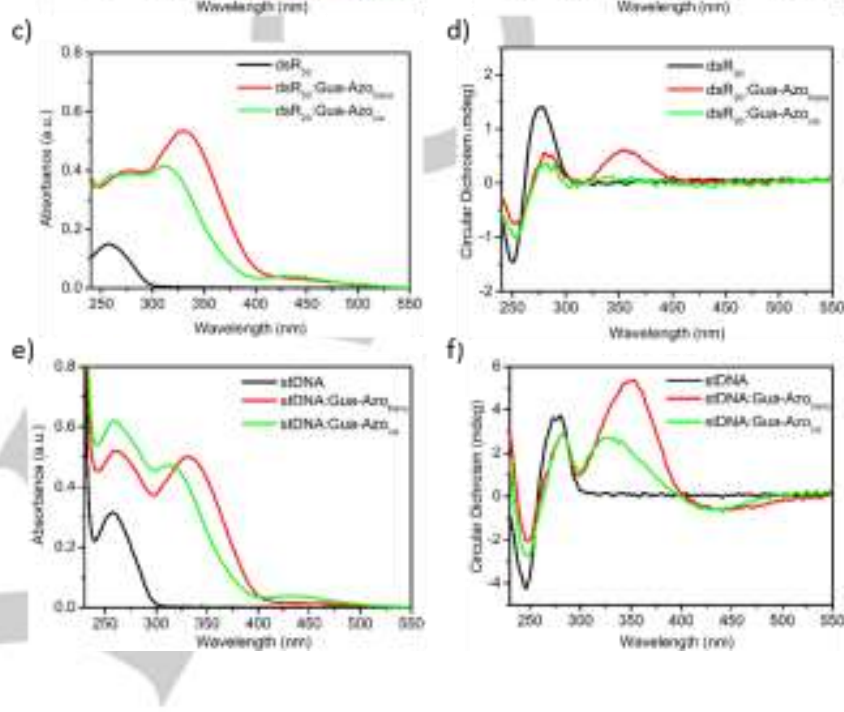

Figure 2. Changes in UV-Vis (left) and CD (right) spectra of several DNA/GuaAzo mixtures at $20^{\circ} \mathrm{C}$ in MQ-Water before (red lines) and after illumination at $365 \mathrm{~nm}$ (green lines): (a-b) $\mathrm{dT}_{40} /$ Gua-Azo at 1:50 molar ratio; (c-d) dsR $20 /$ GuaAzo at 1:50 molar ratio; and (e-f) stDNA

In situ cis $\rightarrow$ trans photoisomerization. In order to use GuaAzo as photo-controllable DNA-binding switch, we considered a cis-trans cycle. A solution of the complex [Gua-Azocis - DNA] was illuminated at $450 \mathrm{~nm}$ (cis-to-trans) for 15 minutes. We observed a red-shifted absorption that coincides with the characteristic band of Gua-Azotrans and with the vanishing of the $n-\pi^{*}$ band (see Figure 3), which is consistent with a trans form of Gua-Azo within the mixture. Interestingly, the ICD from [Gua$\mathbf{A z o}_{\text {trans }}$ - DNA] complex is recovered for the short oligonucleotides upon exposure at $450 \mathrm{~nm}$ (Figure 3b,d), which indicates that the photoisomerization process could be reversed to give the trans isomer. Upon cis-to-trans photoisomerization with stDNA, the bisignate ICD from [Gua-Azo ${ }_{\text {cis }}$. DNA] turns into a positive monosignate signal, red-shifted $\left(\Delta \Lambda_{\max }=27 \mathrm{~nm}\right)$ and with a $\lambda_{\max }$ similar to the one observed for [Gua-Azo trans $_{\text {. DNA] }}$ (Figure 2f, 3f), which indicates that the photoisomerization occurs when the ligand is bound to DNA. In terms of comparison, Baigl et al. pointed out that other guanidinium-azobenzene derivatives possessing either one or two terminal guanidinium groups (with a flexible spacer between the guanidinium group and the azobenzene) also bind via non-covalent interactions with different DNAs. ${ }^{[28]}$ In this case, only the bis-terminated compound exhibited ICD bands in its trans conformation, 
indicating the importance of the geometrical arrangement of the ligands in the assembly with the DNA (see below).

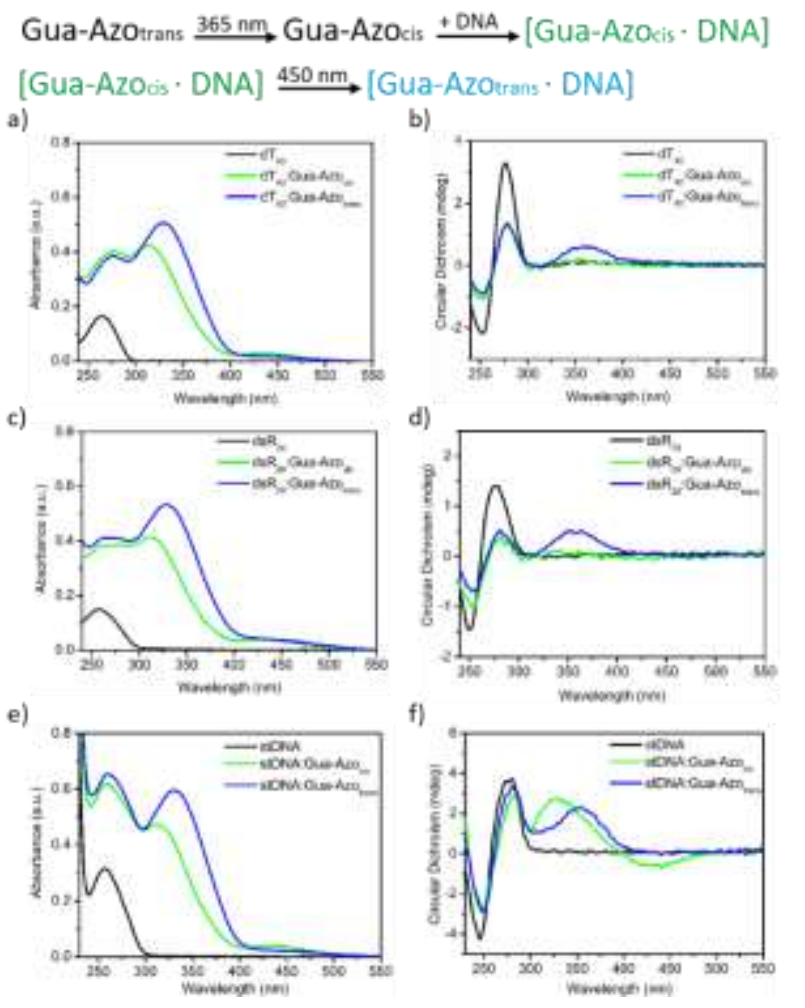

Figure 3. Changes in UV-Vis (left) and CD (right) spectra of several DNA/GuaAzo mixtures at $20{ }^{\circ} \mathrm{C}$ in MQ-Water upon illumination at $365 \mathrm{~nm}$ and at 450 $\mathrm{nm}$ : (a-b) $\mathrm{dT}_{40} /$ Gua-Azo at 1:50 molar ratio; (c-d) dsR $\mathrm{R}_{20} /$ Gua-Azo at 1:50 molar ratio; and (e-f) stDNA $A_{b p} / G u a-A z o$ at 1:1 molar ratio (in base pair/ligand ratio).

In situ trans $\rightarrow$ cis $\rightarrow$ trans photoisomerization. A solution of the complex [Gua-Azotrans - DNA] was illuminated at $365 \mathrm{~nm}$ for 5 minutes. The CD spectra showed the disappearance of the ICD bands, typical of the [Gua-Azocis - DNA] complex (Fig. S18), which indicates that the photoisomerization from trans $\rightarrow$ cis occurred in interaction with DNA (in situ). We then tested a subsequent cis $\rightarrow$ trans photoisomerization in situ, by illuminating the same solution for 15 minutes at $450 \mathrm{~nm}$. We noticed that the ICD signal typical of [Gua-Azotrans . DNA] was not completely recovered, even after longer illumination times (up to $90 \mathrm{~min}$, Fig. S19). Regarding the UV-Vis absorption spectra, we observed that there is lesser extent of red-shift of the maximum absorption band when both photoisomerization processes (trans $\rightarrow$ cis and then cis $\rightarrow$ trans) are realized in interaction with DNA, which may indicate structural changes of the complex after a complete photoisomerisation cycle (see below).

\section{DNA binding modes: docking studies}

We performed docking studies to compare the affinity and the binding modes of Gua-Azo with double-stranded DNA (using a genetic algorithm in the AutoDock4 package on the $\mathrm{dsR}_{20}$ sequence, see protocol in the Supporting Information), for both cis and trans stereoisomers. The most energetically favorable docking solution for each Gua-Azo/DNA complex is depicted in Figure 4. The calculated binding energy is more stable for the [Gua-Azotrans - DNA] complex $\left(E_{b}=-9.99 \mathrm{kcal} / \mathrm{mol}\right)$ than for the [Gua-Azocis - DNA] complex $\left(E_{b}=-8.82 \mathrm{kcal} / \mathrm{mol}\right)$. Although Gua-Azo isomers are both adsorbed in the DNA minor grooves, their binding modes and interactions at play are different, which lead to these differences in stability (see next section), and also on the DNA sequence over which the isomers are adsorbed, as described below for each isomer in these energetically-favorable docking solutions.

Binding modes of trans isomer of Gua-Azo. The nearest DNA bases of $\mathrm{dsR}_{20}$ for Gua-Azotrans (i.e. located at a maximum distance of $4.0 \AA$ A $)$, are $5^{\prime}-. .{ }^{7}$ GTAAATCG $^{14} \ldots-3^{\prime} \quad$ (3'$\ldots{ }^{35}$ GCATTTA ${ }^{29} \ldots-5$ '), see Figure 4 . Remarkably, the shape of Gua-Azo in trans configuration perfectly accommodates the DNA minor-groove, see Figure 4 center in which Gua-Azo is shown in van der Waals spheres. We noticed two main interaction sites between Gua-Azotrans and DNA (Figure 4 left): 1) one strong $\mathrm{H}$-bond between the polar hydrogen of the acylhydrazone group of Gua-Azo ( $\mathrm{HN} 1$ atom, see numbering Figure 4 top) and the nucleobase oxygen of T32 residue (Figure 4a), with optimal $\mathrm{H}$-bond parameters (distance of $2.2 \AA$ and angle of $158^{\circ}$ between donor-hydrogen-acceptor atoms); 2) a pattern of hydrogen-bonds and electrostatic interactions involving the guanidinium moiety with DNA. The HN2 atom is $\mathrm{H}$ bonded to a phosphate oxygen of the $\mathrm{C} 13$ residue (distance 3.5 $\AA$ and angle $150^{\circ}$, see Figure $4 \mathrm{~b}$ ), and HN3 is $\mathrm{H}$-bonded to the sugar oxygen of the same residue, with optimal geometrical parameters of $3.0 \AA$ and $175^{\circ}$ (Figure 4c). Concerning the nitrogen bearing two hydrogen atoms (HN4), one chargeassisted $\mathrm{H}$-bond was detected with the phosphate oxygen of the residue T32 (geometrical parameters: $2.4 \AA$ and $151^{\circ}$, see Figure 4d).

Binding modes of cis isomer of Gua-Azo. The nearest bases of $\mathrm{dsR}_{20}$ for Gua-Azocis are 5'-... ${ }^{10} \mathrm{AATCGGT}{ }^{16} \ldots-3^{\prime}$ (3'$\left..^{33} \mathrm{ATTTAG}^{28} \ldots-5^{\prime}\right)$, i.e. a different preferential sequence site than Gua-Azotrans, see Figure 4. In contrast to the trans stereoisomer, the cis conformation yield to a partial desorption of the azobenzene moiety, as the phenyl at the extremity of GuaAzo is outside the DNA minor-groove (Figure 4 right). The interactions between Gua-Azocis and DNA (see Fig. S20) are: 1) a $\mathrm{H}$-bond between the polar hydrogen of the acylhydrazone group of Gua-Azocis (HN1) and the sugar oxygen of the residue $\mathrm{T} 12$; 2) $\mathrm{H}$-bond between the HN3 hydrogen atom of the guanidinium moiety, and a sugar oxygen of the $A 29$ residue, with non-optimal geometrical parameters (distance $3.1 \AA$, angle $132^{\circ}$, Figure S20). The same hydrogen participates also in $\mathrm{H}$ bonding with a phosphate oxygen of the residue T30, again with non-optimal geometrical parameters $\left(3.3 \AA\right.$ and $\left.145^{\circ}\right)$. Finally, the HN4a hydrogen atom is at a distance of $2.5 \AA$ from an oxygen phosphate of the residue G15. The binding mode of the Gua-Azo $_{\text {cis }}$ isomer can be therefore summarized in two anchor points: one weak $\mathrm{H}$-bond that implicates the $\mathrm{HN} 1$ hydrogen atom, and another anchor point that involves several weak $\mathrm{H}$-bonds of the guanidinium moiety, reinforced by electrostatic interactions. 

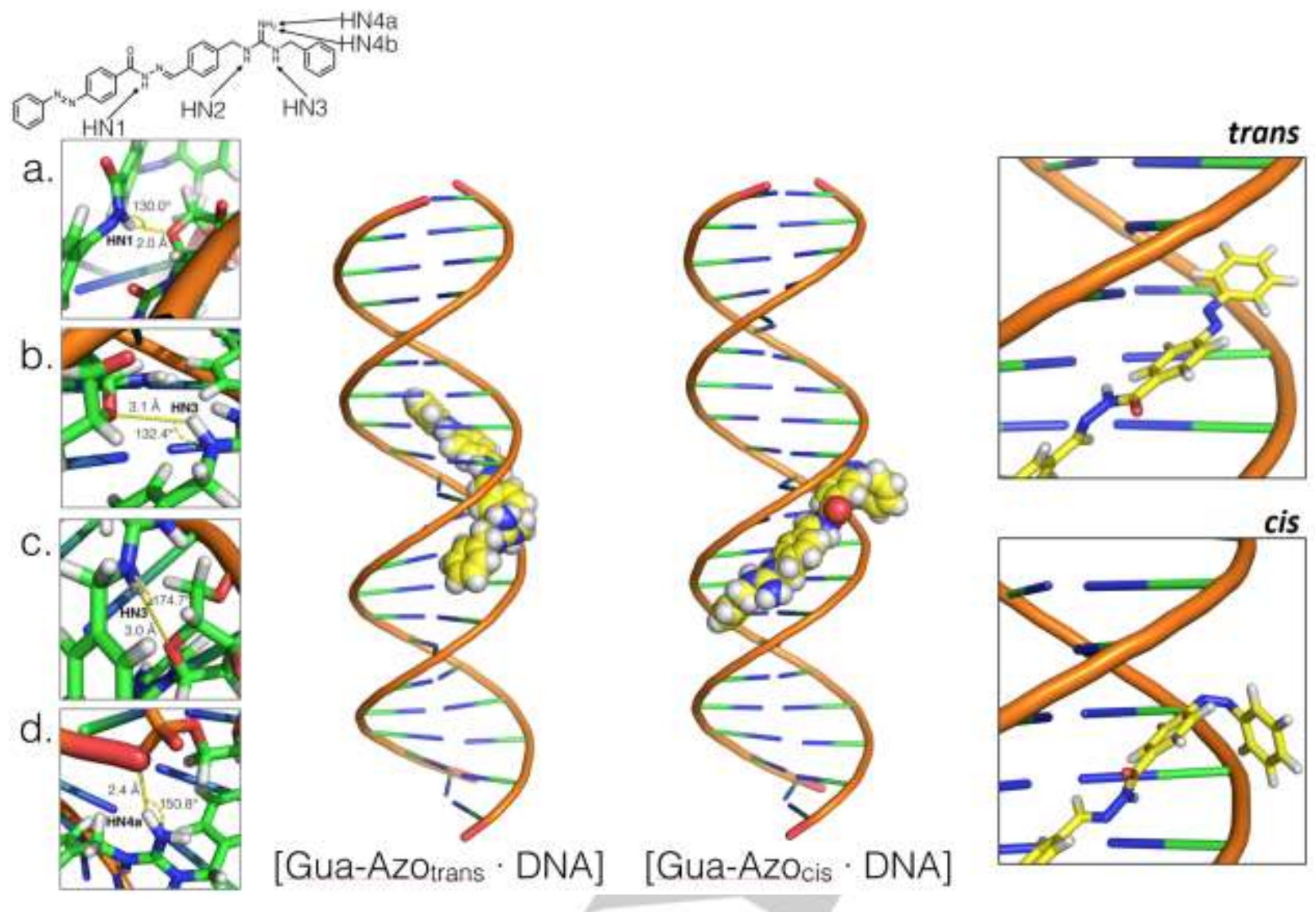

Figure 4. Center: most energetically favorable binding modes for the two GuaAzo isomers with dsR $\mathrm{R}_{20}$ DNA. Center: Gua-Azo is depicted in van der Waals spheres and DNA as ribbons with bases as sticks. Left: close-up views for the main intermolecular interactions for Gua-Azotrans, in stick representation. Right: close-up views at the location of the azobenzene moiety for the two isomers. Water molecules are omitted for clarity.

\section{Stability and dynamics of the complex DNA/photoswitch}

The comparison of the interactions involved in docking solutions of [Gua-Azotrans - DNA] versus [Gua-Azo cis - DNA] indicates that $\mathrm{H}$-bonds are more optimal in terms of directionality in the [GuaAzotrans - DNA] structure, thereby explaining why the GuaAzotrans isomer has a higher affinity with DNA. When considering the ten energetically most favorable complexes obtained from our docking studies for both Gua-Azo isomers, our results show that the trans azobenzene stereoisomer of Gua-Azo is always found to be more stable: the calculated binding energy of the [Gua-Azotrans - DNA] goes from -9.99 to $-9.51 \mathrm{kcal} / \mathrm{mol}$ for the $1^{\text {st }}$ and $10^{\text {th }}$ most stable complex, and from -8.82 to $-7.15 \mathrm{kcal} / \mathrm{mol}$ for the corresponding [Gua-Azocis - DNA] complexes (see Table $\mathrm{S} 2$ in the Supporting Information). Let us note that, for GuaAzotrans, the docking scores for different sequence sites were estimated very close in energy, suggesting many ligands interacting along the same DNA structure, in agreement with the titration experiments. The $10^{\text {th }}$ most stable [Gua-Azotrans - DNA] complex is indeed more stable than the $1^{\text {st }}$ most stable [Gua$\mathbf{A z O}_{\text {cis }}$ - DNA] complex, reinforcing clearly the preferential binding of the Gua-Azotrans isomer to DNA.
The difference of stability between the trans and cis isomers of Gua-Azo when bound to DNA is further shown by DNA melting temperature $\left(T_{m}\right)$ experiments with stDNA, where we monitored the UV-Vis absorbance at $260 \mathrm{~nm}$ of the samples as a function of temperature (Fig. S25). The value of $T_{m}$ is defined as the temperature for which the normalized absorbance equals 0.5 ; this value is $\sim 50{ }^{\circ} \mathrm{C}$ for the pure stDNA in MilliQ water. When a solution of Gua-Azo in trans, this value goes up to $5^{\circ} \mathrm{C}\left(T_{m} \sim\right.$ $55{ }^{\circ} \mathrm{C}$ ), whereas it goes up to $2^{\circ} \mathrm{C}$ when the Gua-Azo was primarily from cis $\left(T_{m} \sim 53^{\circ} \mathrm{C}\right)$. Besides, we noticed that, at temperatures higher than $T_{m}$, the plot for Gua-Azotrans does not show a purely sigmoidal curve, and the $\Delta T_{m}$ between pure stDNA and [Gua-Azotrans - stDNA] further increases. When the same experiment was carried out after an in situ photoisomerisation of the complex, the curves show the same trend than for a non-irradiated mixture of [Gua-AzOtrans - stDNA], with an increase of the melting temperature $\left(T_{m} \sim 59{ }^{\circ} \mathrm{C}\right)$. Altogether, this shows the higher stability of the [Gua-Azotrans . DNA] compared to [Gua-Azo cis $_{\text {· DNA]. }}$ 
To further asses the differences in dynamics between these two states when bound in the DNA minor-grooves, we performed Molecular Dynamics (MD) simulations for the two complexes, starting from coordinates of the best docking poses for $100 \mathrm{~ns}$ MD production (using the AMBER16, see MD methodology in the Supporting Information). For both complexes, a so-called "induced fit" in the DNA minor groove was observed in the beginning of the MD simulations, as shown by the important variation of the RMSD of ligands, followed by a plateau until the end of MD simulations (Fig. S21). In the last part of the MD simulations (90-100 ns), i.e. when the complexes are stabilized, the variation of the RMSD is much higher for Gua-Azocis than for Gua-Azotrans (Fig. S22). The average RMSD and standard deviations over the last 10 ns are 2.4 / $0.4 \AA$ and 2.0 / $0.2 \AA$ for Gua-Azo $_{\text {cis }}$ and Gua-Azotrans, respectively. In addition, the dynamics of [Gua-Azo cis - DNA] and [Gua-Azotrans - DNA] complexes are different. Concerning the acylhydrazone part of the molecule (around HN1), the distances between the HN1 atom and DNA show a normal distribution profile in a narrow range of values, meaning that the central part of ligands is rather static for both complexes (Fig. S23). In contrast, the dynamics of the guanidinium moiety of Gua-Azo are completely different. For the [Gua-Azotrans - DNA] complex, the guanidinium moiety is highly mobile, with an oscillating position between two phosphate groups. The distance between the HN4a atom and DNA presents a two-steps profile and a distribution with two peaks, due to the electrostatic competition between the phosphates of $\mathrm{C} 13$ and T32 DNA residues (Fig. S24). This is not the case for the [Gua-Azocis - DNA] complex, for which the guanidinium group remains static, as shown by the distance profile between $\mathrm{HN} 4 \mathrm{a}$ atom and DNA and its one-peak distribution (Fig. S24). Therefore, the docked Gua-Azo stereoisomers feature different dynamics: Gua-Azotrans has a static azobenzene anchored to the DNA minor groove, and a mobile guanidinium moiety, whereas the azobenzene in Gua$\mathbf{A z O}_{\text {cis }}$ is mobile, flipped outside of the DNA minor groove, with a rather static guanidinium moiety. The static behavior of the GuaAzotrans stereoisomer when bound to DNA, well accommodated with its crescent shape in the DNA minor-groove in a helical manner, is in agreement with the observed induced CD signal in the region where the Gua-Azo absorbs. The numerous possible sites of adsorption along DNA could further explain the intense ICD signals observed. However, a complete identification on the origin of this ICD signal for the trans isomer would need an indepth theoretical analysis (as for other well-known DNA groove binder), ${ }^{[45]}$ which is beyond the scope of this study. In contrast, Gua-Azo $_{\text {cis }}$ stereoisomer is more mobile and less anchored to the DNA, with the azobenzene moiety partially desorbed from the minor-groove, outside the DNA double helix, which could explain the absence of $C D$ signals in the same range.

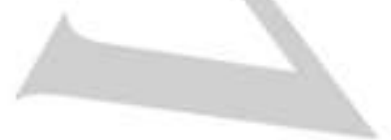

\section{Photoswitching in DNA-templated heteromolecular complexes}

The photo-control of the supramolecular association of Gua-Azo molecules onto DNA templates is attractive to modulate the assembly of other DNA binders. Recently, we have shown that a guanidinium-bispyrene derivative, GuaBiPy (Chart 1), undergoes DNA-templated self-assembly through a combination of interaction with the phosphodiester DNA backbone and $\pi$ stacking between pyrene moieties, with the pyrene moieties adopting a chiral organization within the complex. ${ }^{[11]}$ In order to evaluate the possibility of hetero-molecular complexes of guanidinium derivatives with DNA, mixtures of GuaBiPy and Gua-Azo were prepared in varying proportions but maintaining constant the total concentration of guanidinium compounds (Gua $=$ GuaBiPy + Gua-Azo, the latter either in trans or cis). Each type of DNA was added to these mixtures using the same molar ratio than in the previous experiments, i.e. 1:50 molar ratio for both $\mathrm{dT}_{40}$ : Gua and dsR $\mathrm{R}_{20}$ : Gua, and a 1:1 ratio in base pairs for stDNA:Gua (i.e. one guanidinium derivative per base pair). For mixtures with the oligonucleotides $\left(\mathrm{dT}_{40}\right.$ or $\left.\mathrm{ds}_{20}\right)$, the $C D$ measurements show that, while both pure GuaBiPy and pure Gua-Azotrans interact with $\mathrm{dT}_{40}$ and $\mathrm{ds}_{20}$, yielding ICD signals in the $320-400 \mathrm{~nm}$ range, the ICD signals become less intense than for pure GuaBiPy for the three different component mixtures (GuaBiPy:Gua-Azo 20:80, 50:50, 80:20) (Figs. S2629). This indicates that Gua-Azotrans competes with GuaBiPy in terms of DNA binding. The shape of the ICD signals shows mixed signals between ICD of pure guanidinium derivatives, suggesting the formation of heteromolecular complexes with DNA. A weak ICD signal is maintained for the mixtures with Gua-Azo $_{\text {cis }}$ only for large proportion of GuaBiPy within the mixture (i.e. for 50:50 and 80:20 in GuaBiPy:Gua-Azo). When the [ODN - GuaBiPy - Gua-Azocis] solutions are illuminated in situ at $450 \mathrm{~nm}$ (cis $\rightarrow$ trans), the ICD signals remained weak, and are difficult to interpret.

In contrast, with the long DNA template (stDNA), we observed intense ICD signals for all the mixtures (Figure 5 and Figs. S3031 ), with more intense signals for the $20: 80$ and $50: 50$ GuaBiPy:Gua-Azotrans complexes with stDNA than for the pure stDNA:GuaBiPy or pure stDNA:Gua-Azotrans. This may indicate a cooperative effect in the chiral organization of the ligands when these are interacting with long templates. ${ }^{[46]}$ Upon illuminating a Gua-Azo solution at $365 \mathrm{~nm}$ (trans $\rightarrow$ cis) and then adding GuaBiPy and DNA, the ICD signal of the [stDNA . GuaBiPy - Gua-Azocis] mixture became less intense (except for the 80:20 in GuaBiPy:Gua-Azo, for which it remained constant, Fig. S31h), as the signature of the isomerization of Gua-Azo trans $\rightarrow$ cis. The subsequent in situ illumination of this [stDNA . GuaBiPy - Gua-Azocis] solution at $450 \mathrm{~nm}$ yield ICD signals typical of Gua-Azotrans with stDNA (Fig. S30f), indicative of the cis $\rightarrow$ trans isomerization even when the complex is composed of a mixed assembly along the DNA scaffold. ${ }^{[47]}$ 
a)

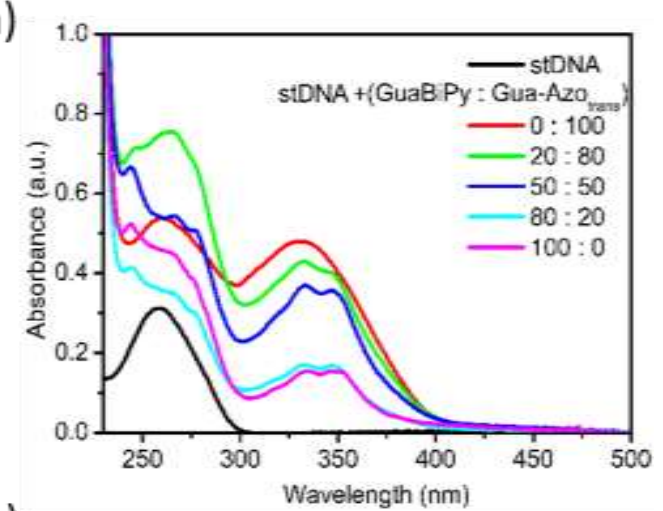

b)

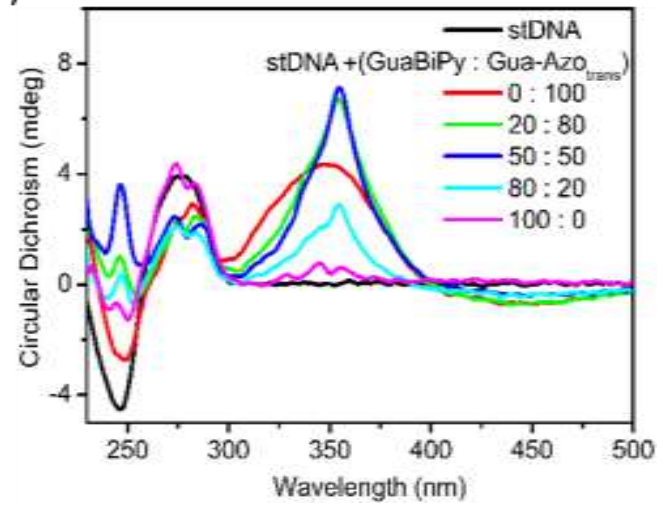

a)

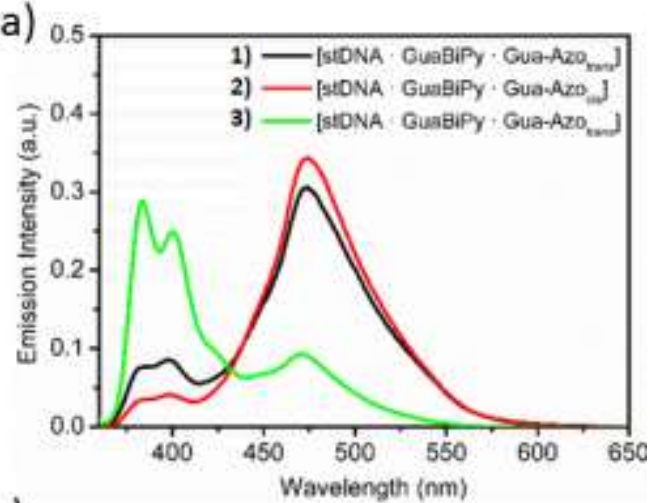

b)

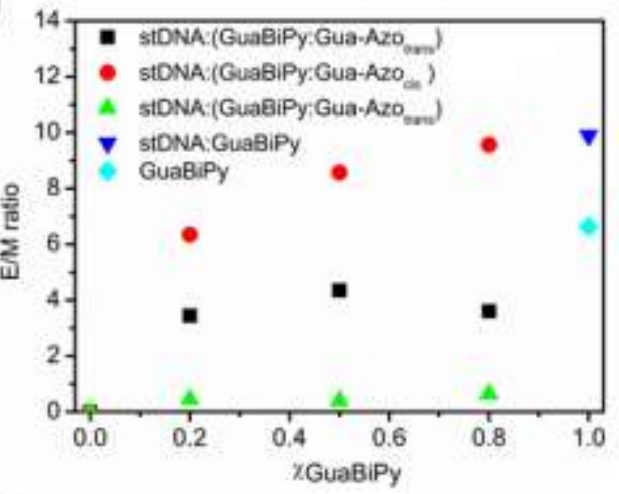

Figure 5. Changes in UV-Vis (a) and CD (b) spectra of several stDNA/GuaBiPy/Gua-Azotrans mixtures (GuaBiPy:Gua-Azo trans 0:100, 20:80, $50: 50,80: 20,100: 0)$ at $20^{\circ} \mathrm{C}$ in Milli-Q Water (Gua-Azo in trans configuration)

To further assess the effect of photoisomerization over the self-assembly of Gua-Azo/GuaBiPy along the DNA template, fluorescence spectroscopy was carried out, as the pyrene moieties are efficient fluorescent probes to assess DNAtemplating effects. It was previously observed in the emission spectra that excimer emission GuaBiPy (maximum at around $470 \mathrm{~nm}$ ) is favoured at the expense of monomer emission (peaking at $396 \mathrm{~nm}$ ) upon interaction with single-stranded DNA, indicative of pure GuaBiPy templated self-assembly, stabilized by $\pi$-stacking of pyrenes. ${ }^{[11,36]}$

In a three-component mixture [ODN - GuaBiPy - Gua-Azo], when Gua-Azo is in trans, the excimer emission is quenched compared to excimer emission of a solution of [ODN - GuaBiPy] see Figs.S33-34. This is further illustrated by plots of excimer/monomer ratio as a function of the composition of GuaBiPy:Gua-Azo in the mixture (Figs. S35). This is also the case for mixtures with the long template [stDNA . GuaBiPy . Gua-Azo], showing a significant decrease in excimer emission with respect to a DNA-templated assembly of pure GuaBiPy (i.e. [stDNA - GuaBiPy]), as observed in E/M ratio of Figure $6 b$ (black squares vs. blue triangle). The weaker excimer emission of pyrenes observed in these three-component mixtures indicates that Gua-Azotrans competes with GuaBiPy in the assembly along the DNA template.
Figure 6. (a) Emission spectra (at $\lambda_{\mathrm{exc}}=348 \mathrm{~nm}$ ) of a mixture of stDNA:GuaBiPy:Gua-Azo (stDNA:ligand: $1: 1$ ratio in base pair, [GuaBiPy] $=[$ Gua-Azo]). [stDNA]bp $=122 \mu \mathrm{M}$ in base pair; [GuaBiPy] + [GuaAzo $]=120 \mu \mathrm{M}$.. (b) Plot of the intensity ratio of excimer emission (taken at $472 \mathrm{~nm}$ ) to monomer emission (taken at $396 \mathrm{~nm}$ ) as a function of the molar ratio in [GuaBiPy]:[Gua-Azo] in the mixtures.

The evolution of emission and spectra excimer/monomer ratio (E/M) for the different mixtures for a complete photoisomerization cycle has been monitored as a function of the composition GuaBiPy:Gua-Azo in the mixture, see Figure 6 (and Figs. S33-36 in the Supporting Information). While the excimer emission of GuaBiPy is lowered for short $\mathrm{dT}_{40}$ templates in presence of Gua-Azo (whatever the isomer of GuaAzo), the situation is different for mixtures with long dsDNA templates (Figure 6a). When going from trans $\rightarrow$ cis, we observed a recovery of the excimer emission of GuaBiPy: the E/M ratio of [stDNA - GuaBiPy - Gua-Azocis] are relatively close to that of pure GuaBiPy with this template, see Figure $6 \mathrm{~b}$ (red

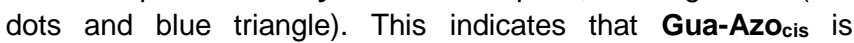
expelled - at least partially - upon trans $\rightarrow$ cis photoisomerization because of lower affinity with dsDNA, and replaced by GuaBiPy, as sketched in Figure 7. When this mixture is then illuminated at $450 \mathrm{~nm}$ (cis $\rightarrow$ trans), the excimer emission is quenched compared to monomer emission ( $E / M$ ratio $<1$ ), which indicates that Gua-Azo in trans replaces GuaBiPy in the templated assembly. Given the highly dynamic behaviour of these DNA-templated self-assemblies, the fact that this process is not fully reversible in terms of $E / M$ ratio for a 
complete photoisomerization cycle (as for ICD signals of [DNA Gua-Azo] complexes, see above) may indicate kinetic processes, with structural changes within the complex from the initial trans to the final trans Gua-Azo configuration after photoisomerization. These structural changes could also originate from DNA damages upon UVA irradiation, ${ }^{[48-49]}$ albeit we used a limited irradiation time ( 5 min with a LED at $365 \mathrm{~nm}$ ) to perform photoisomerization of Gua-Azo with DNA. This is comparable to UVA irradiation times used in other DNA-binding studies with azonbenzene-based peptidomimetic ligand, for which the nativity of DNA was stated to be unchanged. ${ }^{[30]}$

Let us highlight that the E/M ratio of [stDNA - GuaBiPy . Gua-Azotrans] complexes are higher than E/M ratio obtained for complexes with $\mathrm{dT}_{40}$ templates (Figure 6 and Fig. S34), which suggests a better $\pi$-stacked pyrenes arrangement along long dsDNA templates.

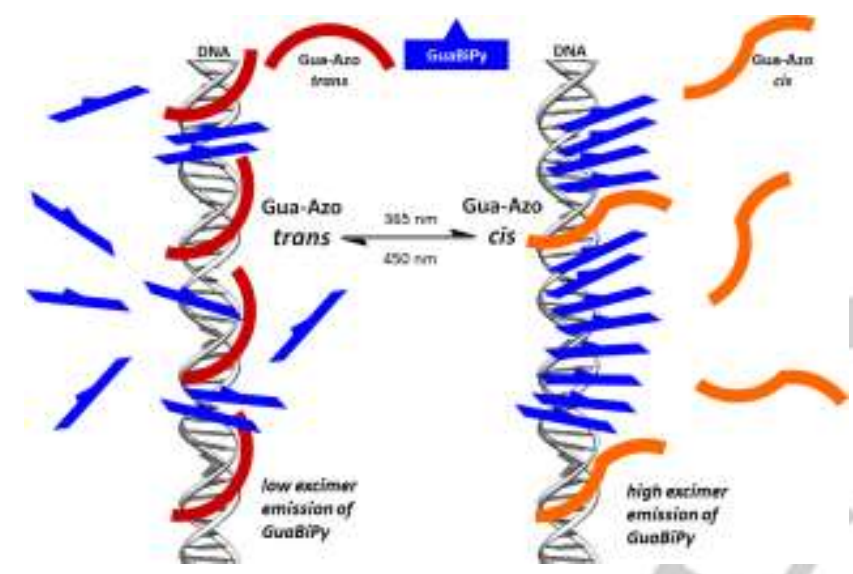

Figure 7. Sketch of the DNA-templated self-assembly of GuaBiPy/GuaAzo depending on the isomerization of Gua-Azo.

\section{Conclusions}

We have reported the design of a photoresponsive DNA ligand, Gua-Azo, which photo-isomerizes between two configurations cis and trans - that have different binding modes and affinities to DNA. We can pinpoint that:

- Gua-Azo binds to the DNA minor groove when the azobenzene moiety is in trans configuration, which is detectable by characteristic chiroptical signals. Gua-Azo is particular in view of recently reported guanidinium-based DNA ligands, ${ }^{[11,32]}$ as it binds to the DNA minor-groove from two sites of interactions (acylhydrazone and guanidinium groups), with a crescent shape that accommodates helically to the DNA minor groove, as shown by docking studies;

- in contrast, the cis configuration is partially desorbed from DNA minor groove and has a lesser affinity towards DNA;

- when Gua-Azo is mixed with another guanidinium-based DNA ligand (GuaBiPy) in presence of DNA, the ligands form heteromolecular supramolecular complexes in a chiral organization along the template;
- the stacking propensity of GuaBiPy along the DNA, reflected by excimer emission of pyrenes, can be indirectly controlled through Gua-Azo photoisomerisation. This amounts to a primitive supramolecular network, ${ }^{[50-53]}$ where the structural photoswitching of one compound has a remote control over the DNA-templated assembly of another compound present in the chemical system, which is detected by unique fluorescence signal output changes.

Altogether, these results show that well-designed photoresponsive DNA binders can be exploited to photo-modulate the supramolecular DNA assembly in templated hetero-molecular complexes. Besides the ability of light-induced ligand redistribution, ${ }^{[54]}$ our approach could be exploited to tune the delivery of competitive small molecules DNA binders and address the dynamics of DNA-templated multicomponent assemblies. This could also serve to photo-manipulate DNA nanostructures (e.g. DNA origami) through a non-covalent, dynamic approach. ${ }^{[5]}$

\section{Experimental Section}

Details of synthesis, characterization, preparation of samples, additional spectra, molecular modelling methodology and analysis, see Supplementary Information at...

\section{Acknowledgements}

The authors thank the COST Action CM 1304 (Emergence and Evolution of Complex Chemical Systems) for a Short Term Scientific Mission (STSM) of J. R.-M. in Montpellier. Research in Mons was supported by the Fonds de la Recherche Scientifique - FNRS under the grants $n^{\circ} 1 . B 333.15 F$ (CHIRNATES) and $n^{\circ}$ F.4532.16 (MIS-SHERPA). This work was also supported by the Science Policy Office of the Belgian Federal Government (BELSPO - PAI 7/5). J.R.-M., M.F., J.K., and M.S. are FNRS researchers. Research in Montpellier was supported by the LabEx CheMISyst (ANR-10-LABX-05-01).

Keywords: DNA-templating • supramolecular self-assembly • photoswitch $\cdot$ minor-groove binder •

[1] J.-P. Sauvage and M. W. Hosseini in Comprehensive Supramolecular Chemistry, Vol. 9, Pergamon (UK), 1996.

[2] H. A. Becerril and A. T. Woolley, Chem. Soc. Rev. 2009, 38, 329.

[3] C. K. McLaughlin, G. D. Hamblin and H. F. Sleiman, Chem. Soc. Rev. 2011, 40, 5647.

[4] a) M. Surin, P. G. A. Janssen, R. Lazzaroni, P. Leclère, E. W. Meije and A. P. H. J. Schenning, Adv. Mater. 2009, 21, 1126. b) A. RuizCarretero, P. G. A. Janssen, A. Kaeser and A. P. H. J. Schenning, Chem. Commun. 2011, 47, 4340. c) J. Lin, M. Surin, D. Beljonne, X. Lou, J. L. J. van Dongen and A. P. H. J. Schenning, Chem. Sci. 2012, 3, 2732.

[5] J. Niu, R. Hili and D. R. Liu, Nat. Chem. 2013, 5, 282.

[6] M. Surin, Polym. Chem. 2016, 7, 4137.

[7] Z.-G. Wang and B. Ding, Adv. Mat. 2013, 25, 3905.

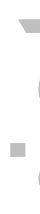


[8] E. Busseron, Y. Ruff, E. Moulin and N. Giuseppone, Nanoscale 2013, 5 7098.

[9] G. Sargsyan, B. M. Leonard, J. Kubelka and M. Balaz, Chem. Eur. J. 2014, 20, 1878.

[10] S. M. D. Watson, M. A. Galindo, B. R. Horrocks and A. Houlton, J. Am. Chem. Soc. 2014, 136, 6649

[11] D. Paolantoni, J. Rubio-Magnieto, S. Cantel, J. Martinez, P. Dumy, M. Surin and S. Ulrich, Chem. Commun. 2014, 50, 14257.

[12] a) P. G. A. Janssen, A. Ruiz-Carretero, D. Gonzalez-Rodriguez, E. W. Meijer and A. P. H. J. Schenning, Angew. Chem. Int. Ed. Engl. 2009, 48 8103. b) Ruiz-Carretero, P. G. Janssen, A. L. Stevens, M. Surin, L. M. Herz and A. P. H. J. Schenning, Chem. Commun. 2011, 47, 884.

[13] X. Yan, F. Wang, B. Zheng and F. Huang, Chem. Soc. Rev. 2012, 41, 6042.

[14] Y. N. Teo and E. T. Kool, Chem. Rev. 2012, 112, 4221

[15] W. Szymański, J. M. Beierle, H. A. V. Kistemaker, W. A. Velema and B. L. Feringa, Chem. Rev. 2013, 113, 6114.

[16] P. Besenius, J. Polym. Sci. A Polym. Chem. 2017, 55, 34

[17] G. Mayer and A. Heckel, Angew. Chem. Int. Ed. 2006, 45, 4900.

[18] M. Balaz, S. Tannir and K. Varga, Coord. Chem. Rev. 2017, 349, 66.

[19] X. Tang and I. J. Dmochowski, Mol. BioSyst. 2007, 3, 100.

[20] C. Garcia-Iriepa, M. Marazzi, L. M. Frutos and D. Sampedro, RSC Adv. 2013, 3, 6241

[21] Y. Yan, X. Wang, J. I. L. Chen and D. S. Ginger, J. Am. Chem. Soc. 2013, 135, 8382

[22] E. Sengupta, Y. Yan, X. Wang, K. Munechika and D. S. Ginger, ACS Nano 2014, 8, 2625.

[23] H. Asanuma, X. Liang, H. Nishioka, D. Matsunaga, M. Liu and M. Komiyama, Nat. Prot. 2007, 2, 203.

[24] A. A. Beharry and G. A. Woolley, Chem. Soc. Rev. 2011, 40, 4422.

[25] H. M. D. Bandara and S. C. Burdette, Chem. Soc. Rev. 2012, 41, 1809.

[26] C. Dugave and L. Demange, Chem. Rev. 2003, 103, 2475.

[27] Y. Nakasone, H. Ooi, Y. Kamiya, H. Asanuma and M. Terazima, J. Am. Chem. Soc. 2016, 138, 9001

[28] H. Asanuma, T. Ito, T. Yoshida, X. Liang and M. Komiyama, Angew. Chem. Int. Ed. 1999, 38, 2393.

[29] X. Liang, T. Mochizuki and H. Asanuma, Small 2009, 5, 1761.

[30] S. Schimka, S. Santer, N. M. Mujkić-Ninnemann, D. Bléger, L. Hartmann, M. Wehle, R. Lipowsky and M. Santer, Biomacromolecules 2016, 17, 1959.

[31] J. Andersson, S. Li, P. Lincoln and J. Andreasson, J. Am. Chem. Soc. 2008, 130, 11836.

[32] A. Bergen, S. Rudiuk, M. Morel, T. Le Saux, H. Ihmels and D. Baigl, Nano Letters 2016, 16, 773.

[33] J. M. Priegue, D. N. Crisan, J. Martinez-Costas, J. R. Granja, F. Fernandez-Trillo and J. Montenegro, Angew. Chem. Int. Ed. 2016, 55, 7492.

[34] E. Bartolami, Y. Bessin, N. Bettache, M. Gary-Bobo, M. Garcia, P. Dumy and S. Ulrich, Org. Biomol. Chem. 2015, 13, 9427.

[35] E. Bartolami, Y. Bessin, V. Gervais, P. Dumy and S. Ulrich, Angew. Chem. Int. Ed. 2015, 54, 10183.
[36] C. Bouillon, D. Paolantoni, J. C. Rote, Y. Bessin, L. W. Peterson, P. Dumy and S. Ulrich, Chem. Eur. J. 2014, 20, 14705.

[37] C. Gehin, J. Montenegro, E.-K. Bang, A. Cajaraville, S. Takayama, H. Hirose, S. Futaki, S. Matile and H. Riezman, J. Am. Chem. Soc. 2013, 135, 9295.

[38] E. I. Geihe, C. B. Cooley, J. R. Simon, M. K. Kiesewetter, J. A. Edward, R. P. Hickerson, R. L. Kaspar, J. L. Hedrick, R. M. Waymouth and P. A. Wender, Proc. Natl. Acad. Sci. USA 2012, 109, 13171.

[39] T. A. Theodossiou, A. Pantos, I. Tsogas and C. M. Paleos, ChemMedChem 2008, 3, 1635

[40] P. A. Wender, W. C. Galliher, E. A. Goun, L. R. Jones and T. H. Pillow, Adv. Drug Delivery Rev. 2008, 60, 452.

[41] E. A. Goun, T. H. Pillow, L. R. Jones, J. B. Rothbard and P. A. Wender, ChemBioChem 2006, 7, 1497.

[42] R. Qi, S. Wu, H. Xiao, L. Yan, W. Li, X. Hu, Y. Huang and X. Jing, J. Mater. Chem. 2012, 22, 18915.

[43] D. Paolantoni, S. Cantel, P. Dumy and S. Ulrich, Int. J. Mol. Sci. 2015, 16, 3609.

[44] E. Bartolami, J. Knoops, Y. Bessin, M. Fossepré, J. Chamieh, P. Dumy M. Surin and S. Ulrich, Chem. Eur. J. 2017, 23, 14323. b) N. Kanfar, A Mehdi, P. Dumy, S. Ulrich, J. Y. Winum, Chem. Eur. J. 2017; c) N. Kanfar, M. Tanc, P. Dumy, C. T. Supuran, S. Ulrich and J. Y. Winum, Chem. Eur. J. 2017, 23, 6788. d) W. Drożdż, C. Bouillon, C. Kotras, S. Richeter, M. Barboiu, S. Clément, A. R. Stefankiewicz and S. Ulrich, Chem. Eur. J. 2017, DOI: 10.1002/chem.201703868.

[45] N. Holmgaard List, J. Knoops, J. Rubio-Magnieto, J. Idé, D. Beljonne, P. Norman, M. Surin and M. Linares, J. Am. Chem. Soc. 2017, 139, 14947-14953.

[46] J. Rubio-Magnieto, M. Kumar, P. Brocorens, J. Idé, S.J. George, R. Lazzaroni and M. Surin, Chem. Comm. 2016, 52, 13873.

[47] Note that the change in the order of addition of GuaBiPy for the [stDNA GuaBiPy.Gua-Azo] mixture (i.e. addition of stDNA to the mixtures of GuaBiPy and Gua-Azo or addition to GuaBiPy directly to the preformed [stDNA:Gua-Azo] complex) forms mixtures with quite similar chiroptical signatures, regardless the Gua-Azo configuration (Fig. S32 in the Supplementary Information).

[48] X. Fang, N. Ide, S.-I. Higashi, Y. Kamei, T. Toyooka, Y. Ibuki, K. Kawai, H. Kasai, K. Okamoto, S. Arimoto-Kobayashi and T. Negishi, Photochem. Photobiol. Sci. 2014, 13, 1338.

[49] E. Sage, P.-M. Girard and S. Francesconi, Photochem. Photobiol. Sci. 2012, 11, 74

[50] N. Giuseppone and J.-M. Lehn, Chem. Eur. J. 2006, 12, 1715.

[51] S. Ulrich and J.-M. Lehn, Chem. Eur. J. 2009, 15, 5640.

[52] G. Vantomme, S. Jiang and J.-M. Lehn, J. Am. Chem. Soc. 2014, 136, 9509.

[53] J.-M. Lehn, Angew. Chem. Int. Ed. 2015, 54, 3276.

[54] D. V. Berdnikova, T. M. Aliyeu, T. Paululat, Y. V. Fedorov, O. A Fedorova and H. Ihmels, Chem. Commun. 2015, 51, 4906.

[55] J. Brglez, P. Nikolov, A. Angelin and C. M. Niemeyer, Chem. Eur. J. 2015, 21, 9440-9446. 
